\title{
FEYNMAN RULES FOR WEYL SPINORS WITH MIXED DIRAC AND MAJORANA MASS TERMS
}

\author{
V. Dūdènas and T. Gajdosik \\ Department of Theoretical Physics, Faculty of Physics, Vilnius University, Sauletekio 9, LT-10222 Vilnius, Lithuania \\ E-mail: vytautasdudenas@inbox.lt; tgajdosik@yahoo.com
}

Received 23 May 2016; revised 8 July 2016; accepted 23 September 2016

\begin{abstract}
We present a basic formalism for using the Weyl spinor notation in Feynman rules. We focus on Weyl spinors with mixed Dirac and Majorana mass terms. To clarify the definitions we derive the Feynman rules from the path integral and present two examples: loop corrections for a fermion propagator and a tree level analysis of a seesaw toy model.
\end{abstract}

Keywords: Weyl spinors, Feynman rules, seesaw mechanism

PACS: $14.60 . S t, 14.60 . P q$

\section{Introduction}

Despite the tremendous success of the Standard Model (SM), there is no doubt that it cannot be a complete theory due to numerous experimental evidences for which the SM fails to find an explanation. One of these experimental evidences is the observation of neutrino oscillations; for a short review of these experiments see references [1], 2]. The oscillations prove that at least two of the neutrinos have masses, but the original assumptions of the SM forbid these mass terms. So the neutrino sector and possibly the Higgs sector should be extended with some new degrees of freedom, i. e. new particles, to allow for the possibility that neutrinos have a mass.

The simplest "building blocks" for a fermionic particle content are Weyl spinors. Thus the model building is usually done in the Weyl spinor notation. However, if one looks at the standard textbooks on quantum field theory (QFT), like [3, 4], one can see that it is unusual to find a proper treatment for Weyl spinors. This is in contrast to supersymmetry (SUSY) references [5, 6 . As a result, non-SUSY calculations using Weyl spinors are somewhat absent in the literature, although the Weyl spinor formalism is known for an easier implementation on computer algebra systems [7]. This is not very surprising as we have only Dirac mass terms in the SM: the 4-component spinor formulation is way easier to deal with in this case. But considering a possible Majorana neutrino [8], theories with mixed Majorana and Dirac mass terms for fermions become relevant; for a review of seesaw mechanisms see [9]. Then the usual 4-component spinor techniques are not so transparent to understand the dynamics of mass mixing, whereas the Weyl spinor notation gives a natural diagrammatic approach to these cases, as we will see in Subsection 4.4 .

The difficulty of using Weyl spinors also arises from having many possibilities of different conventions. We present the definitions, which are essential to understand these possibilities in Section 2. With the conventions from 作 we rederive Feynman rules in order to make these conventions visible by using the path integral approach in Section 3. We focus on the examples that are relevant for studying a seesaw model. This includes loop corrections for a Majorana particle in Subsubsection 3.4.2 and Subsection 4.3 and a diagrammatic approach of the seesaw itself in Subsection 4.4.

\section{Weyl spinors}

\subsection{Definitions}

The Weyl spinor [10, 11] is the fundamental representation of the group SU(2). The Lorentz group is homomorphic to $\mathrm{SU}(2)_{L} \otimes \mathrm{SU}(2)_{R}[12$, where $L$ and $R$ are labels to distinguish the two subgroups. Particles fall into representations of these groups. A particle which is in the fundamental representation of the $L$ subgroup and in the trivial representation of the $R$ subgroup is called a "left-handed" spinor and has left 
chirality. The opposite is true for the "right-handed" spinor.

The two subgroups of the Lorentz group are related by Hermitian conjugation and by parity transformation [12]. So if we do a Hermitian conjugation or a parity transformation on a left-handed field, we get the field in the right-handed representation. Both of the two transformations flip the representations $L \leftrightarrow R$, but the Hermitian conjugation also makes a charge conjugation. For more detailed discussion see [11].

A Weyl spinor is anticommuting, so the symbols that give the spinor metric are antisymmetric. Denoting spinor indices by Latin letters, we write for lefthanded spinors

$$
\xi \chi \equiv \epsilon^{b a} \xi_{a} \chi_{b}
$$

where $\epsilon$ is the totally antisymmetric symbol, which takes a left-handed spinor into its dual space by the definition [5, 13]

$$
\xi^{a} \equiv \epsilon^{a b} \xi_{b}, \xi_{a} \equiv \epsilon_{a b} \xi^{b}, \epsilon^{a b} \epsilon_{b c} \equiv \delta_{c}^{a},
$$

where $\delta$ is the Kronecker symbol. Note that the components of the spinor are Grassmannian (i. e. they anticommute). The Hermitian conjugation puts a spinor into the opposite handiness. The right-handed spinor index is written as a Latin letter with a dot, so $\left(\xi^{a}\right)^{\dagger} \equiv \xi^{\dagger \dot{a}}$. Doing Hermitian conjugation of the scalar product of left-handed spinors

$$
(\xi \chi)^{\dagger} \equiv\left(\epsilon^{b a} \xi_{a} \chi_{b}\right)^{\dagger}=\left(\xi^{b} \chi_{b}\right)^{\dagger}=\chi_{b}^{\dagger} \xi^{\dagger \dot{b}}
$$

and defining the raising and lowering of a right-handed index in a similar way as for the left-handed spinors

$$
\xi^{\dot{a}} \equiv \epsilon^{\dot{a} \dot{b}} \xi_{\dot{b}}, \xi_{\dot{a}} \equiv \epsilon_{\dot{a} \dot{b}} \xi^{\dot{b}}, \epsilon_{\dot{a} \dot{b}} \epsilon^{\dot{b} \dot{c}} \equiv \delta_{\dot{a}}^{\dot{c}},
$$

we can write the definition of this metric:

$$
\epsilon^{12}=\epsilon^{\mathrm{i} \dot{\mathrm{i}}}=\epsilon_{21}=\epsilon_{\mathrm{i \textrm {i }}}=1 \text { and } \epsilon^{21}=\epsilon^{\mathrm{i} \mathrm{i}}=\epsilon_{12}=\epsilon_{\mathrm{ij}}=-1 .
$$

Since the fields anticommute, we get

$$
\xi \chi \equiv \xi^{a} \chi_{a}=-\chi_{a} \xi^{a}=-\epsilon^{a c} \epsilon_{a b} \chi^{b} \xi_{c}=\chi^{a} \xi_{a}=\chi \xi
$$

We define a summation convention for left-handed (undotted) spinor indices to sum from up to down. The Hermitian conjugation reverses this summation, hence dotted indices are summed from down to up; to conclude,

$\xi \chi=\chi \xi \equiv \xi^{a} \chi_{a}=\chi^{a} \xi_{a}, \xi^{\dagger} \chi^{\dagger}=\chi^{\dagger} \xi^{\dagger} \equiv \xi_{\dot{a}}^{\dagger} \chi^{\dagger \dot{a}}=\chi_{\dot{a}}^{\dagger} \xi^{\dagger \dot{a}}$

\subsection{Basic properties and the Lagrangian for Weyl} spinors

The four components of a 4-vector can be written in the space of the direct product $\mathrm{SU}(2)_{L} \otimes \mathrm{SU}(2)_{R^{*}}$. Since the fundamental representation of $S U(2)$ has 2 degrees of freedom, a 4-vector can be seen as the product of two fundamental representations of $S U(2)$, i. e. two Weyl spinors. Hence we can find a connection that transforms two spinors from these two Lorentz's subgroups into a four-component vector in the Minkowski spacetime. The connection is

$$
\xi^{\dagger} \bar{\sigma}^{\mu} \chi=\xi_{\dot{a}}^{\dagger \dagger} \bar{\sigma}^{\mu \dot{a} a} \chi_{a}=-\chi^{a} \sigma_{a \dot{a}}^{\mu} \xi^{\dot{a}}=-\chi \sigma^{\mu} \xi^{\dagger}
$$

with the definition

$$
\bar{\sigma}^{\mu a \dot{a}}=\epsilon^{a b} \epsilon^{\dot{a} \dot{b}} \sigma_{b \dot{b}}^{\mu} .
$$

These connections can be written as

$$
\bar{\sigma}^{\mu \dot{a} a}=(I,-\vec{\sigma})^{\dot{a} a}, \sigma_{a \dot{a}}^{\mu} \equiv(I, \vec{\sigma})_{\dot{a} a},
$$

where $\vec{\sigma}$ is a 3 -vector of Pauli matrices, and $I$ is the $2 \times 2$ identity matrix. The product in Eq. (8) is a 4 -vector composed of two Weyl spinors. If we multiply it with some other 4-vector, we will get a Lorentz scalar,

$$
\begin{aligned}
& \xi^{\dagger}(A \cdot \bar{\sigma}) \chi=\xi_{\dot{a}}^{\dagger} A_{\mu} \bar{\sigma}^{\mu \dot{a} a} \chi_{a}=\epsilon_{\dot{a} \dot{b}} \xi^{\dot{b}} A_{\mu} \bar{\sigma}^{\mu \dot{a} a} \epsilon_{a b} \chi^{b} \\
& =-\chi^{b} A_{\mu} \sigma_{b \dot{b}}^{\mu} \xi^{\dagger \dot{b}}=-\chi(A \cdot \sigma) \xi^{\dagger},
\end{aligned}
$$

where in the first and the last equality the summation convention is being used, which holds for these sigma symbols as well. Assuming $A_{\mu}$ represents a vector field, Eq. (11) forms a valid spinor-vector interaction term in a Lagrangian.

Free field terms in the Lagrangian must be bilinear and Hermitian. Given Eq. (7), it is easy to write down the mass term for a single Weyl spinor. We can write the mass term for a single left-handed spinor $\xi^{L}$ as

$$
\mathcal{L}_{\mathrm{M}}=-\frac{1}{2} M\left(\xi^{L} \xi^{L}+\xi^{L^{\dagger}} \xi^{L \dagger}\right) .
$$

The parameter $M$ is made real by absorbing its phase in the Weyl spinor. The term defined by Eq. (12) is called a Majorana mass term. A single Weyl spinor with such a mass term is called a Majorana particle, since from this Weyl spinor one can construct a four-component Majorana spinor. The factor of $\frac{1}{2}$ is conventional, to avoid additional numerical factors in amplitudes due to 
the symmetry of this coupling. Another possible invariant mass term can couple two Weyl spinors:

$$
\mathcal{L}_{\mathrm{D}}=-m_{D} \xi^{k \dagger} \chi^{L}-m_{D}^{\dagger} \chi^{L \dagger} \xi^{R}
$$

These terms are called Dirac mass terms. If two Weyl spinors share a Dirac mass term and do not have a Majorana mass term, they are usually combined into one Dirac spinor, which is nothing more than two Weyl spinors with the same mass. Such particles are called Dirac particles. It is also possible for Weyl spinors to have all those mass terms, with the result that the Weyl spinors are not in their mass eigenstates. In this case, the diagonalized mass matrix in general will give two different masses for two different Weyl spinors. Those are often called two Majorana particles, since the diagonalized mass terms can be written as in Eq. (12). The difference between Dirac and Majorana fermions is discussed in [11].

From Eq. (11) we see how a vector connects to spinors. The partial derivative $\partial_{\mu}$ is also a vector. So we can write Eq. (11) with $\partial_{\mu}$ in place of the vector to form the kinetic term

$$
\mathcal{L}_{\mathrm{K}}=\mathrm{i} \xi^{L \dagger} \bar{\sigma}^{\mu} \partial_{\mu} \xi^{L},
$$

which is Hermitian up to a total derivative, which does not affect dynamics:

$$
\mathcal{L}_{\mathrm{K}}=\mathrm{i} \xi^{L \dagger} \bar{\sigma}^{\mu} \partial_{\mu} \xi^{L}=\mathrm{i} \xi^{L} \sigma^{\mu} \partial_{\mu} \xi^{L \dagger}+\text { total derivative. }
$$

Note the chirality structure of this term. If we recover indices, we see that

$$
\bar{\sigma}^{\mu \dot{a} a} \partial_{\mu} \xi_{a}^{L}=\psi^{\dot{a}}
$$

has only a dotted index, which means that acting on a spinor with $\sigma \partial$ or $\bar{\sigma} \partial$ gives a spinor that has the opposite chirality than the spinor the operators were acting on.

The superscript $L$ that we used in $\xi^{L}$ and $\chi^{L}$ is just a name of the field. We stick to this convention for naming left-handed spinors with superscript $L$ and righthanded spinors with superscript $R$ that correspond to particles and not to antiparticles. So $\xi^{L \dagger}$ is in the righthanded representation, but it is purely our convention that we call $\xi^{L \dagger}$ an antiparticle. All the results that are obtained for the charge conjugated left-handed spinor apply for a right-handed spinor and vice versa. The chirality is all what matters in taking care of the algebra in this formulation. Hence, keeping track of indices without suppressing them is often useful in order to make less mistakes. Whenever we do not use spinor indices, recall the summation conventions shown in Eq. (7).
A lot of spinor algebra relations consistent with these definitions can be found in [5]. For our purpose, we only need

$$
\begin{aligned}
& {\left[\sigma^{\mu} \bar{\sigma}^{v}+\sigma^{v} \bar{\sigma}^{\mu}\right]_{\alpha}^{\beta}=2 g^{\mu v} \delta_{\alpha}^{\beta},} \\
& {\left[\bar{\sigma}^{\mu} \sigma^{v}+\bar{\sigma}^{v} \sigma^{\mu}\right]_{\dot{\beta}}^{\dot{\alpha}}=2 g^{\mu v} \delta_{\dot{\beta}}^{\dot{\alpha}} .}
\end{aligned}
$$

The spinor indices are suppressed using the summation convention of Eq. (7). $g^{\mu v}$ is the usual Minkowski metric, taken to be diag $(1,-1,-1,-1)$. When connecting these symbols with spinor indices, one can connect only barred to unbarred sigmas. As we will see, this knowledge helps in choosing the right rule for writing amplitudes.

\section{Propagators}

When dealing with the path integral formulation, it is convenient to go to the momentum space. For this we need to define Fourier transformations of the fields. For the left-handed Weyl spinor $\xi^{L}$ we define

$$
\xi^{L}(x)=\int_{k} \mathrm{e}^{-\mathrm{i} k x} \xi^{L}(k), \xi^{L \dagger}(x)=\int_{k} \mathrm{e}^{\mathrm{i} k x} \xi^{L \dagger}(k),
$$

where

$$
\int_{k} \equiv \int \frac{\mathrm{d}^{D} k}{(2 \pi)^{D}}, \int_{x} \equiv \int \mathrm{d}^{D} x,
$$

and $D$ is the number of spacetime dimensions. In the spirit of dimensional regularization $D$ is set to 4 at the end of the calculations. The Dirac delta function in $D$ dimensions is represented by the integral

$$
(2 \pi)^{D} \delta\left(k-k^{\prime}\right)=\int_{x} \mathrm{e}^{-\mathrm{i} x\left(k-k^{\prime}\right)} .
$$

Using Eqs. (19) and (21) in the action $S=\int_{x} \mathcal{L}_{K^{\prime}}$ with $\mathcal{L}_{\mathrm{K}}$ given in Eq. (14), we arrive at the action in the momentum space:

$$
S_{\mathrm{K}}=\int_{x} \mathrm{i}^{L \dagger}(x)(\bar{\sigma} \cdot \partial) \xi^{L}(x)=\int_{p} \xi^{L \dagger}(p)(\bar{\sigma} \cdot p) \xi^{L}(p) .
$$

The Majorana mass term in the momentum space becomes

$$
\begin{aligned}
& S_{\mathrm{M}}=\int_{x}\left\{M \xi^{L}(x) \xi^{L}(x)+M^{\dagger} \xi^{L \dagger}(x) \xi^{L \dagger}(x)\right\} \\
& =\int_{p}\left\{M \xi^{L}(-p) \xi^{L}(p)+M^{\dagger} \xi^{L \dagger}(-p) \xi^{L \dagger}(p)\right\} .
\end{aligned}
$$

For a Dirac particle, where we have 2 Weyl spinors, we could define $L$ and $R$ fields to Fourier transform in the same way. Then we would arrive at a Fourier transformed action, where all fields are expressed in the same $p$ direction. But to have the same appearance 
of the $p$ dependence as in the Majorana case, we rather keep a definition for all left-handed fields the same, i. e. we require $\xi^{R \dagger}$ to Fourier transform the same as $\xi^{L}$, and define

$$
\xi^{R}(x)=\int_{k} \mathrm{e}^{\mathrm{i} i k x} \xi^{R}(k), \quad \xi^{R \dagger}(x)=\int_{k} \mathrm{e}^{-\mathrm{i} k x} \xi^{R \dagger}(k) .
$$

For two Weyl spinors $\xi^{L}$ and $\xi^{R}$, sharing the same Dirac mass, we have the momentum dependences

$$
\begin{aligned}
& S_{\mathrm{D}}=\int_{p}\left\{\xi^{L \dagger}(p)(\bar{\sigma} \cdot p) \xi^{L}(p)+\xi^{R \dagger}(-p)(\sigma \cdot p) \xi^{R}(-p)\right. \\
& \left.-m_{\mathrm{D}} \xi^{R \dagger}(-p) \xi^{L}(p)-m_{D}^{\dagger} \xi^{R}(-p) \xi^{L \dagger}(p)\right\} .
\end{aligned}
$$

Note that we do not have the freedom of choosing the definition of the Fourier transformation in the Majorana case, since we have twice less degrees of freedom.

Eq. (25) can also be written in an alternative form as is evident from Eq. (15). We have

$$
\begin{aligned}
& \int_{p} \xi^{L \dagger}(p)(\bar{\sigma} \cdot p) \xi^{L}(p)=-\int_{p} \xi^{L}(p)(\sigma \cdot p) \xi^{L \dagger}(p) \\
& =+\int_{p} \xi^{L}(-p)(\sigma \cdot p) \xi^{L \dagger}(-p) .
\end{aligned}
$$

Using Eq. (26), the action of Eq. (25) can be written as

$$
\begin{aligned}
& S_{\mathrm{D}}=\int_{p}\left\{\xi^{L}(-p)(\sigma \cdot p) \xi^{L \dagger}(-p)+\xi^{R}(p)(\bar{\sigma} \cdot p) \xi^{R \dagger}(p)\right. \\
& \left.-m_{\mathrm{D}} \xi^{R \dagger}(-p) \xi^{L}(p)-m_{\mathrm{D}}^{\dagger} \xi^{R}(-p) \xi^{L \dagger}(p)\right\} .
\end{aligned}
$$

As we will see, the fact that we can write the kinetic term for a single Weyl spinor in two different ways (Eqs. (25) and (27)) results in the freedom of choosing one of two rules for a single propagator line.

We introduce source functions to the Lagrangian density in the position space as

$$
J^{L}(x) \xi^{L}(x)+J^{R}(x) \xi^{R}(x)+\text { H.c. }
$$

and we define the Fourier transformation of the source functions for left and right fields:

$$
J^{L}(x)=\int_{k} \mathrm{e}^{+\mathrm{i} k x} J^{L}(k), J^{R}(x)=\int_{k} \mathrm{e}^{-\mathrm{i} k x \xi^{R}}(k) .
$$

The Fourier-transformed version of Eq. (28) then becomes

$$
\begin{aligned}
& J^{L}(p) \xi^{L}(p)+J^{R}(-p) \xi^{R}(-p)+J^{L \dagger}(p) \xi^{L \dagger}(p) \\
& +J^{R \dagger}(-p) \xi^{R \dagger}(-p) .
\end{aligned}
$$

The definition for the derivation with respect to the source function is

$$
\begin{aligned}
& \frac{\delta J_{a}\left(p_{1}\right)}{\delta J_{b}\left(p_{2}\right)}=\delta\left(p_{1}-p_{2}\right) \delta_{a}^{b}, \\
& \frac{\delta J_{\dot{a}}^{\dagger}\left(p_{1}\right)}{\delta J_{\dot{b}}^{\dagger}\left(p_{2}\right)}=\delta\left(p_{1}-p_{2}\right) \delta_{\dot{a}}^{b}, \quad \frac{\delta J_{a}\left(p_{1}\right)}{\delta J_{\dot{b}}^{\dagger}\left(p_{2}\right)}=0 .
\end{aligned}
$$

Since all Weyl spinors anticommute, this is true for the sources as well, i. e. $\left\{J^{a}, J^{b}\right\}=\left\{J^{a}, J^{\dagger \dot{b}}\right\}=\left\{J^{\dagger \dot{a}}, J^{\dagger \dot{b}}\right\}=0$. It also holds for their derivatives.

\subsection{Propagator definitions}

A propagator is a 2-point correlation function. Given the path integral $Z(J)=\int[D \phi] \mathrm{e}^{\mathrm{i} S()}$, where $[D \phi]$ stands for a formal measure of all possible field configurations, and the action $S(J)=\int_{x}(\mathcal{L}+J \phi)$, the 2-point correlation function of some scalar field $\phi$ is given by

$$
\begin{aligned}
& \langle 0|\phi(x) \phi(y)| 0\rangle=Z^{-1}(J=0) \int[D \phi] \phi(x) \phi(y) \mathrm{e}^{\mathrm{i} S(J=0)} \\
& =\left.Z^{-1}(J) \int[D \phi] \frac{\delta}{\mathrm{i} \delta J(y)} \frac{\delta}{\mathrm{i} \delta J(x)} \mathrm{e}^{\mathrm{i} S(J)}\right|_{J=0} .
\end{aligned}
$$

Since the correlation functions are evaluated at vanishing sources and the path integral is a function of sources, we abbreviate

$$
Z \equiv Z\left(J, J^{\dagger}\right) \text { and } Z\left|\equiv Z\left(J, J^{\dagger}\right)\right|_{J=J^{\dagger}=0} .
$$

Modifying the propagator definition for Weyl spinors poses some complications mainly because of their anticommutativity properties. We consider a left-handed spinor $\xi$ with an effective action $S\left(J, J^{\dagger}\right)$ $=\int_{x}\left(\mathcal{L}+J^{a} \xi_{a}+\xi_{\dot{a}}^{\dagger} J^{a}\right)$. The product of $J \xi=\xi J$ is invariant, but there is an ambiguity in the sign if we differentiate with respect to the source function. Since we defined $+J^{a} \xi_{a}$ in the action, we have the property

$$
\frac{\delta}{\delta J^{a}}\left(J^{b} \xi_{b}\right)=-\left(J^{b} \xi_{b}\right) \frac{\overleftarrow{\delta}}{\delta J^{a}}=\xi_{a}
$$

where the arrow indicates the direction of acting. This arrow is introduced in order to compare the definitions with [5], where this opposite direction of acting for source derivatives is frequently used. Remembering the summation convention for dotted indices, we have

$$
\frac{\delta}{\delta J_{\dot{a}}}\left(J_{\dot{b}}^{\dagger} \xi^{\dagger \dot{b}}\right)=-\left(J_{b}^{\dagger} \xi^{\dagger \dot{b}}\right) \stackrel{\overleftarrow{\delta}}{\delta J_{\dot{a}}^{\dagger}}=\xi^{\dagger \dot{a}} .
$$

Given this, one can relate the definitions for propagators using source derivatives acting only from the left, with the definitions for propagators given in [5]. They are: 


$$
\begin{aligned}
& \left\langle 0\left|\xi^{\dagger \dot{a}}(x) \xi^{a}(y)\right| 0\right\rangle=Z^{-1} \frac{\delta}{i \delta J_{a}(y)} \frac{\delta}{i \delta J_{\dot{a}}^{\dagger}(x)} Z \mid \\
& =Z^{-1} \frac{\delta}{i \delta J_{\dot{a}}(x)} Z \frac{\overleftarrow{\delta}}{i \delta J_{a}^{\dagger}(y)} \mid \\
& \left\langle 0\left|\xi_{a}(x) \xi_{\dot{a}}^{\dagger}(y)\right| 0\right\rangle=Z^{-1} \frac{\delta}{i \delta J^{\grave{a}}(y)} \frac{\delta}{i \delta J^{a}(x)} Z \mid \\
& =Z^{-1} \frac{\delta}{i \delta J^{a}(x)} Z \frac{\overleftarrow{\delta}}{i \delta J^{\dagger \dot{a}}(y)} \mid, \\
& \left\langle 0\left|\xi_{a}(x) \xi^{b}(y)\right| 0\right\rangle=Z^{-1} \frac{\delta}{i \delta J_{b}(y)} \frac{\delta}{i \delta J^{a}(x)} Z \mid \\
& =Z^{-1} \frac{\delta}{i \delta J^{a}(x)} Z \frac{\overleftarrow{\delta}}{i \delta J_{b}(y)} \mid, \\
& \left\langle 0\left|\xi^{\dagger \dot{a}}(x) \xi_{\dot{b}}^{\dagger}(y)\right| 0\right\rangle=Z^{-1} \frac{\delta}{i \delta J^{\dagger \dot{b}}(y)} \frac{\delta}{i \delta J_{\dot{a}}^{\dagger}(x)} Z \mid \\
& =Z^{-1} \frac{\delta}{i \delta J_{\dot{a}}(x)} Z \frac{\overleftarrow{\delta}}{i \delta J^{\dot{b}}(y)} Z \mid .
\end{aligned}
$$

We use the full definition for propagators, where all corrections for a propagator are encoded in the path integral $Z$. The definitions for propagators in [5] are presented in the free field theory only, but the structure is the same. The only difference is the factor $Z^{-1}$ in front of the expression, which we need to include to keep the right normalization of correlation functions in the presence of higher order corrections.

\subsection{Propagator in momentum space}

Since the momentum space is natural for Feynman diagram calculations, we will define the Fouriertransformed version of the previous propagator expression.

First consider a propagator for the left-handed field $\xi$ of the form

$$
\begin{aligned}
& \left\langle 0\left|\xi_{a}(x) \xi^{b}(y)\right| 0\right\rangle=\left(\int[D \xi] \mathrm{e}^{\mathrm{i} S}\right)^{-1} \int[D \xi] \xi_{a}(x) \xi^{b}(y) \mathrm{e}^{\mathrm{i} S} \\
& =Z^{-1} \frac{\delta}{\mathrm{i} \delta J_{b}(y)} \cdot \frac{\delta}{\mathrm{i} \delta J^{a}(x)} Z \mid .
\end{aligned}
$$

We first take a look how derivatives with respect to source functions transform under Fourier transformations. Making use of the Fourier transformations defined in Eq. (19) and the chain rule for functional derivatives, which is just a generalization of $\frac{\partial}{\partial x_{i}}=\sum_{j} \frac{\partial y_{j}}{\partial x_{i}} \frac{\partial}{\partial y_{j}}$, we get

$$
\begin{aligned}
& \frac{\delta}{\delta J^{L}(x)}=\int \mathrm{d}^{D} p \frac{\delta J^{L}(p)}{\delta J^{L}(x)} \frac{\delta}{\delta J^{L}(p)} \\
& =\int_{p}(2 \pi)^{D} \frac{\int_{x_{1}} \mathrm{e}^{-i p x_{1}} \delta J^{L}\left(x_{1}\right)}{\delta J^{L}(x)} \frac{\delta}{\delta J^{L}(p)}=\int_{p} \mathrm{e}^{-i p x_{1}} \frac{(2 \pi)^{D} \delta}{\delta J^{L}(p)}
\end{aligned}
$$
have

One can check that for the opposite chirality we

$$
\frac{\delta}{\delta J^{L \dagger}(x)}=\int_{p} e^{+i p x} \frac{(2 \pi)^{D} \delta}{\delta J^{L \dagger}(p)}
$$

Putting Eq. (41) into Eq. (40) we get

$$
\begin{aligned}
& \left\langle 0\left|\xi_{a}(x) \xi^{b}(y)\right| 0\right\rangle=Z^{-1} \frac{\delta}{\mathrm{i} \delta J_{b}(y)} \cdot \frac{\delta}{\mathrm{i} \delta J^{a}(x)} Z \\
& =Z^{-1} \int_{p, p^{\prime}} \mathrm{e}^{-\mathrm{i} p x} \mathrm{e}^{-\mathrm{i} p^{\prime} y} \frac{(2 \pi)^{D} \delta}{\mathrm{i} \delta J_{b}\left(p^{\prime}\right)} \cdot \frac{(2 \pi)^{D} \delta}{\mathrm{i} \delta J^{a}(p)} Z .
\end{aligned}
$$

The propagator depends only on the spacetime difference $x-y$ and not on $x$ and $y$ separately. So the propagator should Fourier transform with a single factor of $x-y$. By rearranging exponents from Eq. (43) and adjusting the signs of the momentum to have the $\mathrm{e}^{-\mathrm{i} p(x-y)}$ factor in front, we get

$$
\begin{aligned}
& \left\langle 0\left|\xi_{a}(x) \xi^{b}(y)\right| 0\right\rangle=\int_{p} \mathrm{e}^{-\mathrm{i} p(x-y)} \\
& \times\left[Z^{-1} \int_{p^{\prime}} \mathrm{e}^{-\mathrm{i} y\left(p-p^{\prime}\right)} \frac{(2 \pi)^{D} \delta}{\mathrm{i} \delta J_{b}\left(-p^{\prime}\right)} \cdot \frac{(2 \pi)^{D} \delta}{\mathrm{i} \delta J^{a}(p)} Z \mid\right] .
\end{aligned}
$$

This expression still depends on the spacetime point $y$, because we did not yet restrict the coordinate space propagator to depend only on the spacetime difference $x-y$. But the translational invariance of the action always gives this spacetime dependence for correlation functions, hence the correlation function is a translational invariant itself. To preserve this symmetry we need to have $p^{\prime}=p$ in the momentum space; the additional exponent in the brackets of Eq. (44) will just give the identity all the time. There is also an additional integration $\int_{p}$, which might seem strange at first glance. But actually, this integration is what is needed to set $p^{\prime}=p$. To understand this, consider that we have two derivatives with respect to sources. Since the sources are set to zero, the terms that contribute from the action must also come as bilinear functions of sources. After differentiation with respect to the sources we should have two Dirac delta functions, recalling the definition of differentiation in Eq. (31). Because we have an action in the momentum space as $\int_{\omega} \mathcal{L}(\omega)$, we have only one integration measure coming 
from the action, which uses one Dirac delta function to fix one momentum. So the integration $\int_{p^{\prime}}$ is needed to fix the other free momentum. Hence $p^{\prime}$ serves just as a dummy integration variable that matches dimensions and sets $p^{\prime}=p$ at the end of the algebra. As a result of these considerations, we can safely omit the exponent in the brackets $\left(\mathrm{e}^{-\mathrm{i} y\left(p-p^{\prime}\right)} \rightarrow 1\right)$ from our definitions for propagators. Furthermore, since we always have $p^{\prime}=p$ in the end, there is no difference on what source function we put this integration variable: we can change $\frac{(2 \pi)^{D} \delta}{\mathrm{i} \delta J_{b}\left(-p^{\prime}\right)} \cdot \frac{(2 \pi)^{D} \delta}{\mathrm{i} \delta J^{a}(p)} \rightarrow \frac{(2 \pi)^{D} \delta}{\mathrm{i} \delta J_{b}(-p)} \cdot \frac{(2 \pi)^{D} \delta}{\mathrm{i} \delta J^{a}\left(p^{\prime}\right)}$ in Eq. (44) without any consequence. However, the signs of the momenta depend on the choice of the Fourier transformation.

We define Fourier transformations for 2-point correlation functions to be

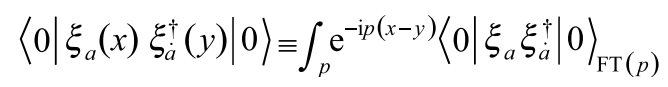

and

$$
\left\langle 0\left|\xi_{a}(x) \xi^{b}(y)\right| 0\right\rangle \equiv \int_{p} \mathrm{e}^{-\mathrm{i} p(x-y)}\left\langle 0\left|\xi_{a} \xi^{b}\right| 0\right\rangle_{\mathrm{FT}(p)},
$$

where $\operatorname{FT}(p)$ labels that the Fourier transformed version of $\left\langle 0\left|\xi_{a}(x) \xi^{b}(y)\right| 0\right\rangle$ depends only on the momentum $p$. The propagators in Eqs. (36) and (39) have an opposite chirality structure compared to those of Eqs. (45) and (46). We defined that fields that are of opposite chirality to each other transformed with the opposite momentum sign in Eq. (19). In order to be consistent with this definition, we have Fourier transformations for the propagators in Eqs. (36) and (39) with the opposite momentum sign relative to Eqs. (45) and (46). This is also consistent with momentum dependences in the free field actions of Eqs. (22), (23), (25) and (27). Given these definitions, we get the expressions for all four types of propagators:

$$
\begin{aligned}
& \left\langle 0\left|\xi^{a^{\dagger}} \xi^{a}\right| 0\right\rangle_{\mathrm{FT}(p)}=Z^{-1} \int_{p^{\prime}} \frac{(2 \pi)^{D} \delta}{\mathrm{i} \delta J_{a}\left(p^{\prime}\right)} \cdot \frac{(2 \pi)^{D} \delta}{\mathrm{i} \delta J_{a}^{\dagger}\left(p^{\prime}\right)} Z \mid \\
& \left\langle 0\left|\xi_{a} \xi_{a}^{\dagger}\right| 0\right\rangle_{\mathrm{FT}(p)}=Z^{-1} \int_{p^{\prime}} \frac{(2 \pi)^{D} \delta}{\mathrm{i} \delta J^{\dagger \dot{a}}(-p)} \cdot \frac{(2 \pi)^{D} \delta}{\mathrm{i} \delta J^{a}\left(-p^{\prime}\right)} Z \mid, \\
& \left\langle 0\left|\xi_{a} \xi^{b}\right| 0\right\rangle_{\mathrm{FT}(p)}=Z^{-1} \int_{p^{\prime}} \frac{(2 \pi)^{D} \delta}{\mathrm{i} \delta J_{b}(-p)} \cdot \frac{(2 \pi)^{D} \delta}{\mathrm{i} \delta J^{\mathrm{a}}\left(p^{\prime}\right)} Z \mid, \\
& \left\langle 0\left|\xi^{\dagger a} \xi_{b}^{\dagger}\right| 0\right\rangle_{\mathrm{FT}(p)}=Z^{-1} \int_{p^{\prime}} \frac{(2 \pi)^{D} \delta}{\mathrm{i} \delta J^{\dagger \dot{b}}(p)} \cdot \frac{(2 \pi)^{D} \delta}{\mathrm{i} \delta J_{\dot{a}}^{\dagger}\left(-p^{\prime}\right)} Z \mid
\end{aligned}
$$

Now we take two Weyl spinors, $\xi^{L}$ and $\xi^{R}$, with the same Dirac mass that couples them together.
Since we introduced a Fourier transform in such a way that $\xi^{L}$ transforms the same as $\xi^{R^{\dagger}}$, we can already write propagators for this Dirac particle by just relabelling fields and without changing momentum dependences:

$$
\begin{aligned}
& \left\langle 0\left|\xi^{L+a} \xi^{L a}\right| 0\right\rangle_{\mathrm{FT}(p)}=Z^{-1} \int_{p^{\prime}} \frac{(2 \pi)^{D} \delta}{\mathrm{i} \delta J_{a}^{L}(p)} \cdot \frac{(2 \pi)^{D} \delta}{\mathrm{i} \delta J_{\dot{a}}^{L \dagger}\left(p^{\prime}\right)} Z \mid, \\
& \left\langle 0\left|\xi_{a}^{R \dagger} \xi_{a}^{R}\right| 0\right\rangle_{\mathrm{FT}(p)}=Z^{-1} \int_{p^{\prime}} \frac{(2 \pi)^{D} \delta}{\mathrm{i} \delta J^{R \dot{a}}(p)} \cdot \frac{(2 \pi)^{D} \delta}{\mathrm{i} \delta J^{R \dagger a}\left(p^{\prime}\right)} Z \mid, \\
& \left\langle 0\left|\xi^{L b} \xi_{a}^{R^{\dagger \dagger}}\right| 0\right\rangle_{\mathrm{FT}(p)}=Z^{-1} \int_{p^{\prime}} \frac{(2 \pi)^{D} \delta}{\mathrm{i} \delta J^{R+a}(-p)} \cdot \frac{(2 \pi)^{D} \delta}{\mathrm{i} \delta J_{b}^{L}\left(p^{\prime}\right)} Z \mid, \\
& \left\langle 0\left|\xi_{\dot{b}}^{R} \xi^{L \dagger \dot{a}}\right| 0\right\rangle_{\mathrm{FT}(p)}=Z^{-1} \int_{p^{\prime}} \frac{(2 \pi)^{D} \delta}{\mathrm{i} \delta J_{\dot{a}}^{L \dagger}(-p)} \cdot \frac{(2 \pi)^{D} \delta}{\mathrm{i} \delta J^{R \dot{b}}\left(p^{\prime}\right)} Z \mid .
\end{aligned}
$$

These propagators can be written differently, for example, one can use the propagator $\left\langle 0\left|\xi_{a}^{L} \xi_{\dot{a}}^{L \dagger}\right| 0\right\rangle_{F T(p)}$ instead of $\left\langle 0\left|\xi^{L+a} \xi L a\right| 0\right\rangle_{F T(p)}$. The changes should be clear from Eqs. (47) to (50).

\subsection{Propagator for a free field}

Considering free fields, it is always possible to shift fields in the action in such a way that the field dependent part is separated from the source dependent part. To be more precise, consider we have a field $\xi$ and we shift it to $\xi^{\prime}$ such that the path integral becomes

$$
\begin{aligned}
& Z(J)=\int[D \xi] \mathrm{e}^{\mathrm{i} S(\xi))}=\int[D \xi] \mathrm{e}^{\mathrm{i}\left(\xi^{\prime}\right)+i(S)}=N \mathrm{e}^{\mathrm{is}())}, \\
& N=\int\left[D \xi^{\prime}\right] \mathrm{e}^{\mathrm{i}(\xi)} .
\end{aligned}
$$

The integration over fields gives just a constant factor $N$ to the path integral $Z(J)$.

Now let us consider the possible shift for Weyl spinors. We use left- and right-handed fields $\xi^{L}$ and $\xi^{R}$ sharing a Dirac mass term. Then the fields are shifted by a linear combination of sources, i. e. the left-handed field will be shifted by a linear combination of left- and right-handed sources. From Subsection 2.2 we know that constructing something that is left-handed from an originally right-handed spinor can be done with $\sigma p$. It is easier to see this if we restore spinor indices. When two spinors have only a Dirac mass, the shift for the left-handed field will have the form

$$
\xi_{a}^{L}(p) \rightarrow \xi_{a}^{L}(p)+x \cdot(\sigma p)_{a \dot{a}} J^{R \dot{a}}(-p)+y \cdot J_{a}^{L}(p),
$$

where $x$ and $y$ are just some unknown constants. The minus sign in the momentum dependence comes from the fact that the propagation of the right-handed field in the negative time direction is the left-handed field in the positive time direction. The important 
thing is keeping track of the chirality. Having in mind our labelling of $R$ and $L$, the shift for $\xi^{R}$ is the same as for $\xi^{L}$ except for interchanging the labels $R \leftrightarrow L$ and the connection $\sigma \rightarrow \bar{\sigma}$ due to the opposite chirality of $\xi^{R}$. In the case of one left-handed spinor with the Majorana mass only, we can identify $R \rightarrow L \dagger$ in Eq. 56).

It is possible to calculate the coefficients $x$ and $y$ by straightforwardly inserting the shift, Eq. (56), into the action and requiring terms that couple sources with fields to cancel. However, since this form of the shift includes the transformation between the left and right chiral states, it makes sense to combine the left and right chiral states into one 2-component vector, where the components are Weyl spinors. This is just the usual 4-component spinor in the chiral representation. How this is done one can find in the appendix of [5]. The source dependent part of the action for two Weyl spinors sharing a Dirac mass term is

$$
\begin{aligned}
& \mathrm{i} S(J)=-\int_{p}\left\{J^{L \dagger}(p) \frac{\mathrm{i}(\bar{\sigma} \cdot p)}{p^{2}-m_{\mathrm{D}}^{2}} J^{L}(p)+J^{R \dagger}(-p) \frac{\mathrm{i}(\sigma \cdot p)}{p^{2}-m_{\mathrm{D}}^{2}} J^{R}(-p)\right. \\
& \left.+\frac{\mathrm{i} m_{\mathrm{D}}^{\dagger}}{p^{2}-m_{\mathrm{D}}^{2}} J^{R \dagger}(-p) J^{L}(p)+\frac{\mathrm{i} m_{\mathrm{D}}}{p^{2}-m_{\mathrm{D}}^{2}} J^{R}(-p) J^{L \dagger}(p)\right\} .
\end{aligned}
$$

Remembering the definitions of propagators in Eqs. (51) to (54) we get

$$
\begin{aligned}
& \left\langle 0\left|\xi^{L \dagger} \xi^{L}\right| 0\right\rangle_{\mathrm{FT}(p)}=\mathrm{i} \frac{\bar{\sigma} \cdot p}{p^{2}-m_{\mathrm{D}}^{2}}, \\
& \left\langle 0\left|\xi^{R \dagger} \xi^{R}\right| 0\right\rangle_{\mathrm{FT}(p)}=\mathrm{i} \frac{\sigma \cdot p}{p^{2}-m_{\mathrm{D}}^{2}}, \\
& \left\langle 0\left|\xi^{L} \xi^{R \dagger}\right| 0\right\rangle_{\mathrm{FT}(p)}=\mathrm{i} \frac{m_{\mathrm{D}}^{\dagger}}{p^{2}-m_{\mathrm{D}}^{2}}, \\
& \left\langle 0\left|\xi^{R} \xi^{L \dagger}\right| 0\right\rangle_{\mathrm{FT}(p)}=\mathrm{i} \frac{m_{\mathrm{D}}}{p^{2}-m_{\mathrm{D}}^{2}} .
\end{aligned}
$$

Comparing Eq. (57) with Eqs. (25) and (27), we see that the same action can be written as

$$
\begin{aligned}
& \mathrm{i} S(J)=-\int_{p}\left\{J^{L}(-p) \frac{\mathrm{i}(\sigma \cdot p)}{p^{2}-m_{\mathrm{D}}^{2}} J^{L \dagger}(-p)+J^{R}(p) \frac{\mathrm{i}(\bar{\sigma} \cdot p)}{p^{2}-m_{\mathrm{D}}^{2}} J^{R \dagger}(p)\right. \\
& \left.+J^{L}(p) \frac{\mathrm{i} m_{\mathrm{D}}^{\dagger}}{p^{2}-m_{\mathrm{D}}^{2}} J^{R \dagger}(-p)+J^{R}(-p) \frac{\mathrm{i} m_{\mathrm{D}}}{p^{2}-m_{\mathrm{D}}^{2}} J^{L \dagger}(p)\right\} \cdot(62)
\end{aligned}
$$

Eqs. (58) and (59) can be written in alternative forms by changing $p \rightarrow-p$ and exchanging $\sigma \leftrightarrow \bar{\sigma}$. Hence we conclude that in this notation

$$
\frac{\mathrm{i} \bar{\sigma} \cdot p}{p^{2}-m_{\mathrm{D}}^{2}} \text { is equivalent to } \frac{-\mathrm{i} \sigma \cdot p}{p^{2}-m_{\mathrm{D}}^{2}} \text {. }
$$

If we have a Weyl spinor with a Majorana mass term, the action can be written as

$$
\begin{aligned}
& \mathrm{i} S(J)=-\frac{1}{2} \int_{p}\left\{J^{L \dagger}(p) \frac{\mathrm{i}(\bar{\sigma} \cdot p)}{p^{2}-M^{2}} J^{L}(p)+J^{L}(-p) \frac{\mathrm{i}(\sigma \cdot p)}{p^{2}-M^{2}} J^{L \dagger}(-p)\right. \\
& \left.+\frac{\mathrm{i} M}{p^{2}-M^{2}} J^{L}(-p) J^{L}(p)+\frac{\mathrm{i} M}{p^{2}-M^{2}} J^{L \dagger}(-p) J^{L \dagger}(p)\right\} .
\end{aligned}
$$

It is obvious that the same equivalence for propagators shown in Eq. (63) holds too. Using this action we get only two independent propagators instead of four, but all four forms, as seen from Eqs. (47) to (50), are present.

\subsection{Propagator for the interacting theory}

In the previous section we saw the free field terms of the action. If we consider an interacting theory, we have an additional term $S_{\text {int }}$ and the path integral becomes

$$
Z \sim \mathrm{e}^{\mathrm{i} \mathrm{S}_{\text {int }}+\mathrm{i}_{\text {free }}}=\mathrm{e}^{\mathrm{i} \mathrm{S}_{\text {int }}} \mathrm{e}^{\mathrm{i} \mathrm{S}_{\text {free }}} .
$$

Most of the models in particle physics are built to describe the interactions as a perturbative series of this expression. The only case when the perturbation theory is not applicable is when we have a bound state. Since we are interested in models that should describe interactions with neutrinos (which do not participate in such states), treating $\mathrm{i}_{\text {int }}$ as a perturbation is general enough. The free field term is expressed in terms of the source functions, so the interaction term then can be expressed as derivatives with respect to sources acting on the free field action: $S_{\text {int }}$ is promoted to an operator $\hat{S}_{\text {int }}$. Then the path integral becomes

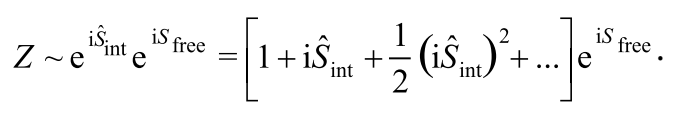

Given the Lagrangian of a theory and using the definitions for propagators of Eqs. (47) to (50) or Eqs. (51) to (54), we can calculate corrections for the tree level propagators to the desired order. The first term of this expansion is just a free field approximation that we discussed in the previous subsection. The second term becomes zero after setting sources to zero as will become clear after we work out the expressions for $\mathrm{i} \hat{S}_{\text {int }}$. The third term in the expansion gives a loop correction for the propagators. In the following subsection we summarize the expressions of $i \hat{S}_{\text {int }}$ for possible interactions with Weyl spinors. 


\subsubsection{Vertices}

We will consider possible couplings that appear in renormalizable models in four dimensions. We will leave, however, the letter $D$ in the exponents of phase space integrals, denoting the number of dimensions as a free parameter in our expressions. This emphasizes that dimensional regularization can be used before setting $D=4$.

In four dimensions a spinor can couple to a vector or a scalar. The spinor-vector coupling term in the action is

$$
\begin{aligned}
& \mathrm{i} S_{i n t}^{V}=+\mathrm{i} g \int_{x} \xi^{L}(x)(A(x) \cdot \sigma) \xi^{L^{\dagger}}(x) \\
& =-\mathrm{i} g \int_{x} \xi^{L^{\dagger}}(x)(A(x) \cdot \bar{\sigma}) \xi^{L}(x) .
\end{aligned}
$$

This is the same term written in two different ways, where $g$ is some coupling constant. We promote this term to an operator $i \hat{S}_{\text {int }}^{V}$ by changing the fields to the corresponding derivatives with respect to the sources. By making use of Eqs. (41) and (42) we go to the momentum space. The expressions for $\mathrm{i} \vec{S}_{\text {int }}$ are

$$
\begin{aligned}
& \mathrm{i} \hat{S}_{i n t}^{V}=+\mathrm{i} g \int_{p_{1}, p_{2}, p_{3}}(2 \pi)^{D} \delta\left(p_{1}+p_{2}+p_{3}\right) \sigma_{a \dot{a}}^{\mu} \\
& \times \frac{(2 \pi)^{D} \delta}{\mathrm{i} \delta J_{A}^{\mu}\left(p_{1}\right)} \frac{(2 \pi)^{D} \delta}{\mathrm{i} \delta J_{\dot{a}}^{L \dagger}\left(-p_{3}\right)} \frac{(2 \pi)^{D} \delta}{\mathrm{i} \delta J_{a}^{L}\left(p_{2}\right)}
\end{aligned}
$$

or

$$
\begin{aligned}
& \mathrm{i} \hat{S}_{i n t}^{V}=-\mathrm{i} g \int_{p_{1}, p_{2}, p_{3}}(2 \pi)^{D} \delta\left(p_{1}+p_{2}+p_{3}\right) \bar{\sigma}^{\mu \dot{a} a} \\
& \times \frac{(2 \pi)^{D} \delta}{\mathrm{i} \delta J_{A}^{\mu}\left(p_{1}\right)} \frac{(2 \pi)^{D} \delta}{\mathrm{i} \delta J^{L a}\left(p_{2}\right)} \frac{(2 \pi)^{D} \delta}{\mathrm{i} \delta J^{L \dot{\dagger}(}\left(-p_{3}\right)},
\end{aligned}
$$

where we restored spinor and vector indices. We see that there is a freedom in choosing the connection between the vector and the spinors, i. e. we can choose either $\bar{\sigma}$ or $-\sigma$ to write down the same vertex. The minus sign for the momentum comes from the definition of the Fourier transformation presented in Eqs. (19) and (24).

The spinor scalar coupling can come in two forms:

$$
\frac{1}{2} y_{1} \phi \xi^{L} \xi^{L}+\text { H.c., and } y_{2} \phi \xi^{R \dagger} \xi^{L}+\text { H.c. }
$$

Here the first term couples some scalar $\phi$ to the same Weyl spinor and the second term couples it to two different spinors. We will always introduce the factor $\frac{1}{2}$ in the definition of the coupling of a scalar with two spinors of the same field in order to cancel additional combinatorial factors that appear due to the symmetry of this term. We take $\phi$ to be a complex scalar for generality, so that we have a complex coupling constant.
Using Eq. (41) we get $i \hat{S}_{\text {int }}^{s}$ for the scalar case in terms of the source functions

$$
\begin{aligned}
& \mathrm{i} \hat{S}_{\text {int }}^{S}=\frac{1}{2} \mathrm{i} y_{1} \int_{p_{1}, p_{2}, p_{3}}(2 \pi)^{D} \delta\left(p_{1}+p_{2}+p_{3}\right) \\
& \frac{(2 \pi)^{D} \delta}{\mathrm{i} \delta J_{\phi}\left(p_{1}\right)} \cdot \frac{(2 \pi)^{D} \delta}{\mathrm{i} \delta J^{L a}\left(p_{2}\right)} \cdot \frac{(2 \pi)^{D} \delta}{\mathrm{i} \delta J_{a}^{L}\left(p_{3}\right)}
\end{aligned}
$$

or

$$
\begin{aligned}
& \mathrm{i} \hat{S}_{\mathrm{int}}^{\mathrm{S}}=\mathrm{i} y_{2} \int_{p_{1}, p_{2}, p_{3}}(2 \pi)^{D} \delta\left(p_{1}+p_{2}+p_{3}\right) \\
& \times \frac{(2 \pi)^{D} \delta}{\mathrm{i} \delta J_{\phi}\left(p_{1}\right)} \cdot \frac{(2 \pi)^{D} \delta}{\mathrm{i} \delta J^{R \uparrow a}\left(p_{2}\right)} \cdot \frac{(2 \pi)^{D} \delta}{\mathrm{i} \delta J_{a}^{L}\left(p_{3}\right)} .
\end{aligned}
$$

The Hermitian conjugate of $i \hat{S}_{\text {int }}^{\text {s }}$ just gives the Hermitian conjugate coupling constants and opposite signs for the momentum dependences in the source functions.

\subsubsection{The spinor-vector loop}

Now we can calculate corrections to all propagators defined in Eqs. (51) to (54). We see that the term linear in $\mathrm{i} S_{\text {int }}$ vanishes for a propagator after setting sources to zero, since all possible interaction terms acting on the free field part of the path integral will leave an odd number of sources. Therefore the loop correction comes from $\frac{1}{2}\left(\mathrm{i} \hat{S}_{\text {int }}\right)^{2}$ in Eq. (66). To see how the path integral formalism applies, we work out the example for the loop correction to the propagator defined in Eq. (51).

Consider the loop correction to a propagator for a fermion with only a Majorana mass and a vector boson in the loop. Let us call the spinor $\xi^{L}$ and the vector $A_{\mu}$. The free field term for this fermion is given in Eq. (64). The interaction operator then is either Eq. (69) or Eq. (68). Let us use the form of Eq. (68). Then the interaction operator to order $O\left(g^{2}\right)$ is

$$
\begin{aligned}
& \frac{1}{2}\left(\mathrm{i} \hat{S}_{\mathrm{int}}\right)^{2}=\frac{1}{2}(\mathrm{ig})^{2} \int_{p_{1}, p_{2}, p_{3}, k_{1}, k_{2}, k_{3}}\left(2 \pi p_{3}^{D}+p_{3}\right)(2 \pi)^{D} \delta\left(k_{1}+k_{2}+k_{3}\right) \\
& \times \sigma_{a \dot{a}}^{\mu} \frac{(2 \pi)^{D} \delta}{\mathrm{i} \delta J_{A}^{\mu}\left(p_{2}\right)} \frac{(2 \pi)^{D} \delta}{\mathrm{i} \delta J_{\dot{a}}^{L \dagger}\left(-p_{3}\right)} \frac{(2 \pi)^{D} \delta}{\mathrm{i} \delta J_{a}^{L}\left(p_{1}\right)} \\
& \times \sigma_{b \dot{b}}^{v} \frac{(2 \pi)^{D} \delta}{\mathrm{i} \delta J_{A}^{v}\left(k_{2}\right)} \frac{(2 \pi)^{D} \delta}{\mathrm{i} \delta J_{\dot{b}}^{L^{\dagger}}\left(-k_{3}\right.} \frac{(2 \pi)^{D} \delta}{\mathrm{i} \delta J_{b}^{L}\left(k_{1}\right)} .
\end{aligned}
$$

We abbreviate the fermion propagators of the free field as

$$
\bar{P}(p)=\frac{\mathrm{i} \bar{\sigma} \cdot p}{p^{2}-M^{2}}, P(p)=\frac{\mathrm{i} \sigma \cdot p}{p^{2}-M^{2}}, P_{M}(p)=\frac{\mathrm{i} M}{p^{2}-M^{2}},
$$


suppressing the spinor indices. We will use the letter $G$ as an abbreviation for the boson propagator, which is an even function of the momentum:

$$
G(p)=G(-p) .
$$

Let us take $A_{\mu}$ to be neutral. In this context, it is enough to say that a neutral field is self-conjugate, i. e. it is its own antiparticle. On the Lagrangian level we have a factor of $\frac{1}{2}$ in front of the bilinear terms due to this extra symmetry, hence the free field term for a neutral boson after completing the squares is

$$
\mathrm{i} S=-\frac{1}{2} \int_{p} J(-p) G(p) J(p) .
$$

For the propagator of the $A_{\mu}$ field we write $G_{A \mu \nu}(p)$, which is also symmetric under $\mu \leftrightarrow v$.

With the definition of the propagator in Eq. (51), the correction to one loop order is

$$
\begin{aligned}
& \left\langle 0\left|\xi^{L \dagger \dot{a}} \xi^{L a}\right| 0\right\rangle_{\mathrm{FT}(p)}^{[2]}=Z^{-1} \int_{p^{\prime}} \frac{(2 \pi)^{D} \delta}{\mathrm{i} \delta J_{a}^{L}(p)} \cdot \frac{(2 \pi)^{D} \delta}{\mathrm{i} \delta J_{\dot{a}}^{L \dagger}\left(p^{\prime}\right)} \\
& \times \frac{1}{2}\left(\mathrm{i} \hat{\mathrm{S}}_{\text {int }}\right)^{2} \mathrm{e}^{\mathrm{i} S_{\text {free }} \mid,}
\end{aligned}
$$

where the number in the brackets of the superscript denotes the order of the correction. In Eq. (77) we have 8 derivatives with respect to the sources in total. Since the propagator is evaluated at vanishing sources, only the term $\frac{1}{4 !}\left(\mathrm{is}_{\text {free }}\right)^{4}$ will contribute from the expansion of the free field part of the path integral. Looking at Eq. (73) we see that in Eq. (77) we have derivatives with respect to $3 J^{L \dagger}$ s, $3 J^{L} S$ and $2 J_{A} s$. The only non-vanishing terms are those that have the same number of sources. These contributions from $\frac{1}{4 !}\left(\mathrm{is}_{\text {free }}\right)^{4}$ are

$$
\begin{aligned}
& \int_{\omega_{1}, \omega_{2}, \omega_{3}, \omega_{4}} \frac{4}{4 !} \frac{1}{2} J_{A} G_{A} J_{A}\left(J^{L \dagger} \bar{P} J^{L}\right)^{3} \text { or } \\
& \int_{\omega_{1}, \omega_{2}, \omega_{3}, \omega_{4}} \frac{4 !}{4 !} \frac{1}{2} J_{A} G_{A} J_{A}\left(J^{L \dagger} \bar{P} J^{L}\right) \frac{1}{2}\left(J^{L} P_{M} J^{L}\right) \frac{1}{2}\left(J^{L \dagger} P_{M} J^{L \dagger}\right),
\end{aligned}
$$

where $\omega_{1}, \omega_{2} \ldots$ are the momenta of different pairs of source functions. All the indices are contracted. The momentum dependences of the source functions can be seen in Eqs. (76) and (64). Acting with source derivatives we get 8 Dirac delta functions that are integrated over with the momenta $\omega_{1}, \omega_{2} \ldots$. In the end one arrives at an expression that can be diagrammatically expressed as a Feynman diagram.

To see explicitly how this is done, we take the first term of Eq. (78) as an example:

$$
\begin{aligned}
& \Pi=Z^{-1} \int_{p^{\prime} \mathrm{i} \delta J_{a}^{L}(p)} \frac{(2 \pi)^{D} \delta}{\mathrm{i} \delta J_{\dot{a}}^{L \dagger}\left(p^{\prime}\right)} \\
& \times \frac{1}{2}\left(\mathrm{i} \hat{S}_{\mathrm{int}}\right)^{2} \int_{\omega_{1}, \omega_{2}, \omega_{3}, \omega_{4}} \frac{4}{4 !} \frac{1}{2} J_{A} G_{A} J_{A}\left(J^{L \dagger} \bar{P} J^{L}\right)^{3} .
\end{aligned}
$$

The easiest start is to differentiate this term first with respect to the vector bosons, since it can be done independently: $\frac{(2 \pi)^{D} \delta}{\mathrm{i} \delta J_{A}^{\mu}\left(p_{2}\right)} \frac{(2 \pi)^{D} \delta}{\mathrm{i} \delta J_{A}^{v}\left(k_{2}\right)}$. Remembering the definition of differentiation, Eq. (31), we get

$$
\begin{aligned}
& \frac{(2 \pi)^{D} \delta}{\mathrm{i} \delta J_{A}^{\mu}\left(p_{2}\right)} \cdot \frac{(2 \pi)^{D} \delta}{\mathrm{i} \delta J_{A}^{v}\left(k_{2}\right)} \int_{\omega_{1}} \frac{1}{2} J^{\rho}\left(-\omega_{1}\right) G_{A \rho \sigma}\left(\omega_{1}\right) J^{\sigma}\left(\omega_{1}\right) \\
& =\int_{\omega_{1}}(2 \pi)^{D} \delta\left(p_{2}+\omega_{1}\right) G_{A \mu \nu}\left(\omega_{1}\right)(2 \pi)^{D} \delta\left(\omega_{1}-k_{2}\right) \\
& =(2 \pi)^{D} \delta\left(p_{2}+k_{2}\right) G_{A \mu \nu}\left(k_{2}\right) .
\end{aligned}
$$

Differentiating in the same manner with respect to the spinor sources from Eq. (73) and integrating with respect to the momenta $\omega_{2}, \omega_{3}$, and $\omega_{4}$, we are left with 6 Dirac delta functions in total $(2$ coming from Eq. (73)). The integrations over all momenta, coming from is $S_{\text {int }}$ in Eq. (73), will connect these momenta to preserve momentum conservation. The last integration over $p^{\prime}$ completes it with setting $p^{\prime}=p$ as discussed in Subsection 3.2. П, shown in Eq. (79), becomes the sum of

$$
\begin{aligned}
& B_{1}=\bar{P}^{f f}(p)\left\{+\frac{1}{2}(2 \pi)^{D} \delta(0)(\mathrm{ig})^{2}\right. \\
& \left.\times \int_{p_{1}, p_{2}} \bar{P}^{\dot{a} a}\left(p_{1}\right) \sigma_{a \dot{a}}^{\mu} D_{A \mu \nu}(0) \sigma_{b \dot{b}}^{v} \bar{P}^{\dot{b} b}\left(p_{2}\right)\right\}, \\
& B_{2}=\bar{P}^{\dot{f f}}(p)\left\{-\frac{1}{2}(2 \pi)^{D} \delta(0)(\mathrm{i} g)^{2}\right. \\
& \left.\times \int_{p_{1}, p_{2}} D_{A \mu v}\left(p_{2}\right) \sigma_{a \dot{a}}^{\mu} \bar{P}^{a b}\left(p_{1}+p_{2}\right) \sigma_{b b}^{v} \bar{P}^{\dot{b} a}\left(p_{1}\right)\right\}, \\
& T=-(\mathrm{ig})^{2}\left[\bar{P}^{\dot{e} a}(p) \sigma_{a \dot{a}}^{\mu} \bar{P}^{\dot{a} f}(p)\right] D_{A \mu v}(0) \int_{k}\left[\sigma_{b \dot{b}}^{v} \bar{P}^{\dot{b} b}(k)\right] \text {, }
\end{aligned}
$$

and

$$
\left.L=+\bar{P}^{\dot{f e}}(p)[\operatorname{ig})^{2} \int_{k} \sigma_{e \dot{a}}^{v} \bar{P}^{\dot{a} a}(p+k) \sigma_{a \dot{d}}^{\mu} D_{A \mu \nu}(k)\right] \bar{P}^{d t}(p) .
$$

These terms are represented as Feynman diagrams in Fig. 1. Arrows on the lines show the flow of the left chirality, i. e. they point from dotted to undotted indices. The momentum flow is taken from left to right as shown with additional arrows near the momenta. 
(a)

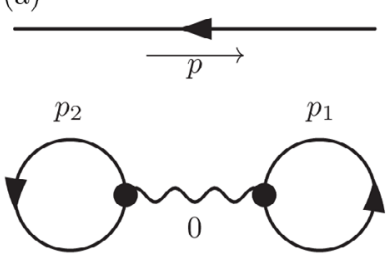

$B_{1}$ in Eq. (81)

(c)

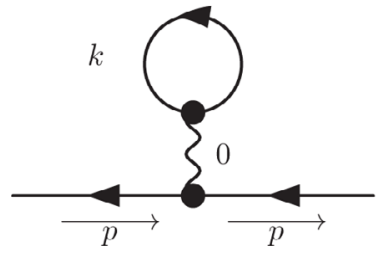

$T$ in Eq. (83) (b)

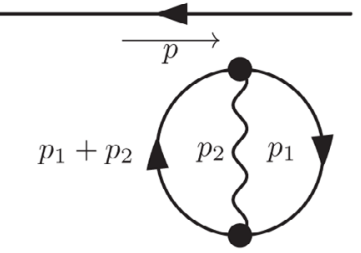

$B_{2}$ in Eq. (82)

(d)

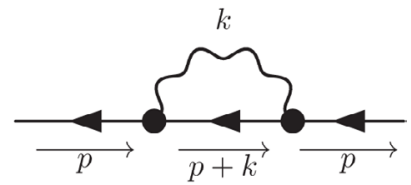

$A$ in Eq. (84)

Fig. 1. Feynman diagrams showing the terms in Eqs. (81) to (84). The bubbles $B_{1}$ and $B_{2}$ shown in (a, b) are cancelled by the normalization of the path integral. $T$, the tadpole connected to the propagator, shown in (c), vanishes. $A$, shown in (d), is the contributing loop correction to the propagator.

The amplitudes in Eqs. (81) and (82) are the socalled vacuum bubbles. They are cancelled by $Z^{-1}$ to all orders. To prove it for this order, see the $O\left(g^{2}\right)$ of $Z$ at vanishing sources. By the same argument of matching the number of derivatives with the number of sources we get

$$
\frac{1}{2}\left(\mathrm{i} \hat{S}_{\text {int }}\right)^{2} \mathrm{e}^{\mathrm{i} S_{\text {free }}} \mid=\frac{1}{2}\left(\mathrm{i} \hat{S}_{\text {int }}\right)^{2} \frac{1}{3 !}\left(\mathrm{i} S_{\text {free }}\right)^{3} .
$$

The only non-vanishing terms from $\frac{1}{2}\left(\mathrm{iS}_{\hat{S}_{\text {in }}}\right)^{2} \frac{1}{3 !}\left(\mathrm{is}_{\text {free }}\right)^{3}$ are

$$
\begin{aligned}
& \frac{1}{2}\left(\mathrm{i} \hat{S}_{\text {int }}\right)^{2} \int_{\omega_{1}, \omega_{2}, \omega_{3}} \frac{3}{3 !} \frac{1}{2} J_{A} D_{A} J_{A}\left(J^{L \dagger} \bar{P} J^{L}\right)^{2} \text { and } \\
& \frac{1}{2}\left(\mathrm{i} \hat{S}_{\text {int }}\right)^{2} \int_{\omega_{1}, \omega_{2}, \omega_{3}} \frac{3 !}{3 !} \frac{1}{2} J_{A} D_{A} J_{A} \frac{1}{2}\left(J^{L} P_{M} J^{L}\right) \frac{1}{2}\left(J^{L \dagger} P_{M} J^{L \dagger}\right) .
\end{aligned}
$$

Working out the first term, one arrives at the terms that are shown in the brackets of Eqs. (81) and (82).

There is also an interesting factor of $\frac{1}{2}$ in Eqs. (81) and (82), which stands for the symmetry factor of these diagrams, i. e. the diagram is identical if you change places of two identical fermion propagators or places of two vertices. Also, there is a minus sign in Eq. (82). This is due to the anticommutativity of fermions: each closed fermion loop gives a relative minus sign to the amplitude. Just as expected, the symmetry factors and the rule for closed fermion loops are the same as in the usual Feynman diagram calculus.

An interesting diagram is drawn from Eq. (83), which is a tadpole connected to a propagator. Note that it has a minus sign due to the closed fermion loop. This already looks strange from a physical perspective: the gauge boson of momentum 0 is vanishing into the vacuum. Since a vector has a Lorentz index, we might worry about the Lorentz invariance if this would contribute. But it does vanish: the propagator $\bar{P}(k)$ is an odd function of $k$, so the term $\int_{k}\left[\sigma_{b \dot{b}}^{v} \bar{P}^{b b}(k)\right]$ gives 0 when integrating over all values of the momentum. Note that the propagator $P_{M}(k)$ is even: a tadpole diagram appearing with this propagator would give a contribution. This cannot happen with a gauge boson, but it appears in the interaction with scalars as will be discussed in Subsection 4.3. So we are left only with Eq. (84) contributing to the one loop correction. (a)

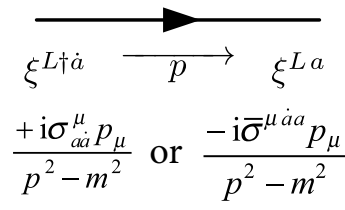

(c)

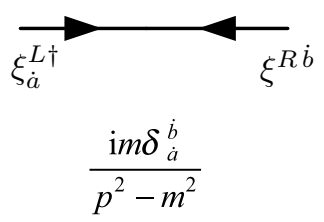

(b)

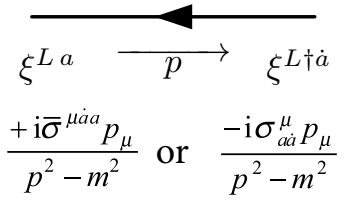

(d)

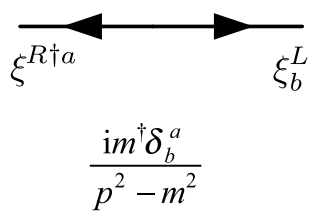

Fig. 2. Feynman diagrams and rules for propagators. These are all possible diagrams for propagators of Weyl spinors. The corresponding Feynman rule is written under each diagram. For the propagators shown in $(a, b)$ one can choose between two possible rules. The mass term can be either Dirac or Majorana in these rules, but for the Majorana case one has to identify $\xi^{R}=\xi^{L \dagger}$ in (c, d). Propagators in (c, d) are even functions of the momentum, hence the direction of the momentum flow is irrelevant and not shown in the diagrams.

\section{Feynman rules}

\subsection{Propagators and mass insertions}

All the definitions in this paper are consistent with the definitions of [阿]. To present Feynman rules for Weyl spinors, one has to include a chirality flow in the diagram. An arrow on the propagator line is defined to show the direction in which the left chirality flows, i. e. the arrow is directed from the dotted index towards the undotted index. Whenever a chiral symmetry breaking term appears (such as a mass), the directions of arrows indicate this by showing opposite directions in the diagram. This is in contrast 
with Feynman diagrams for 4-component spinors, where the direction of an arrow is defined as fermion flow, which has to be preserved all the time in order to have fermion number conservation. Feynman rules for propagators of Weyl spinors are shown in Fig. 2.

We first consider the propagators shown in Fig. 2(a, b). We draw an additional arrow near the propagator line showing the momentum flow. The definition of momentum flow is crucial for these propagators in order to assign the correct rule. To see why this is the case, recall Eq. (63). We have two alternative forms of writing down the expression for the same propagator and this form is related with the direction of momentum. This freedom can be understood comparing the two equivalent expressions for the same action shown in Eqs. (25) and (27) in terms of Weyl spinors or in Eqs. (57) and (62) in terms of sources functions. These alternative forms of writing down the same action are reflected in the rules shown in Fig. 2 (a, b). We can go from one form to the other by either flipping the arrow of the propagator, or by changing the direction of the momentum. For Fig. 2(a), where the left chirality goes from left to right, we have the propagator $\sim \sigma p$ or by changing $p \rightarrow-p$ we have $\sim \bar{\sigma} p$. Equivalently, if we flip the direction of chirality as in Fig. 2(b), we have the propagator $\sim \bar{\sigma} p$ or $\sim-\sigma p$.

The propagators of Eqs. (60) and (61), shown in Fig. 2(c, d), exist only if the mass term is not zero. These propagators are even functions of the momentum, hence the direction of the momentum is not important. Since the mass term for fermions couples different chiral states, the direction of the arrow is not preserved along the propagator line for these propagators.

All the rules for propagators shown in Fig. 2 are obtained using the action of Eq. (57) and the definitions of the propagators from Eqs. (51) to (54). Alternatively, one could start from a chirality preserving action, where the mass terms are zero, and treat the mass terms as couplings. Then we have massless propagators as the first approximation in Fig. 2( $(a, b)$. Taking a Dirac mass term, Eq. (13), as a coupling, we get the Feynman rules shown in Fig. 3 with $m=m_{\mathrm{D}}$. Making an infinite sum of even numbers of mass insertions into the massless propagator for the Weyl spinor, we recover the mass term in the denominators of the propagators shown in Fig. 2( $a, b)$. Making an infinite sum of odd numbers of mass insertions gives rise to the propagators of Fig. 2( $(c, d)$. If we have a Majorana mass as in Eq. (12) instead, we will have just the same rules of Fig. 3 with $m=M$. Making the infinite sums of these insertions will give all the same rules shown in Fig. 2 identifying $\xi^{R}=\xi^{L^{\dagger}}$ and $m=M$. (a)

(b)

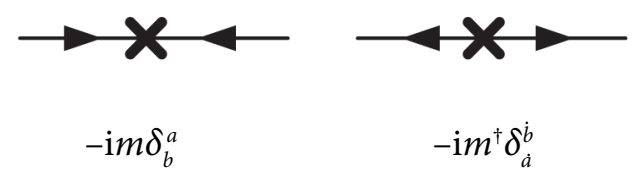

Fig. 3. Mass insertion diagrams and rules. These diagrams correspond to mass terms if they are treated as couplings. These rules can be used for either a Dirac or a Majorana mass term in the same way, i. e. $m=m_{\mathrm{D}}$ if we have a Dirac mass term as in Eq. (13) and $m=M$ if we have a Majorana mass term as in Eq. (12). The direction of arrows shows the chirality structure of the mass term. The momentum conservation along the line is understood. The direction of the momentum flow is irrelevant just as in Fig. 2(c, d).

\subsection{Vertices}

To define the set of rules for interactions with Weyl spinors, one just needs to understand the chirality structure of the interaction terms. The scalar-spinor interaction term changes chirality. Hence the arrows of the spinor lines point in opposite directions in the diagrams as shown in Fig. $4(b, c)$. The momenta are defined to flow into the vertex and the Dirac delta function of these momenta gives the momentum conservation at the vertex. In Fig. 4(b) we define the coupling constant $y$ to come from the term that couples two left-handed spinors as in Eq. (70). Figure 4 (c) is just the Hermitian conjugate of Fig. A (b) with a coupling $y^{\dagger}$. If the scalar field is real, then one can define the phase of the spinors in such a way that $y^{\dagger}=y$.
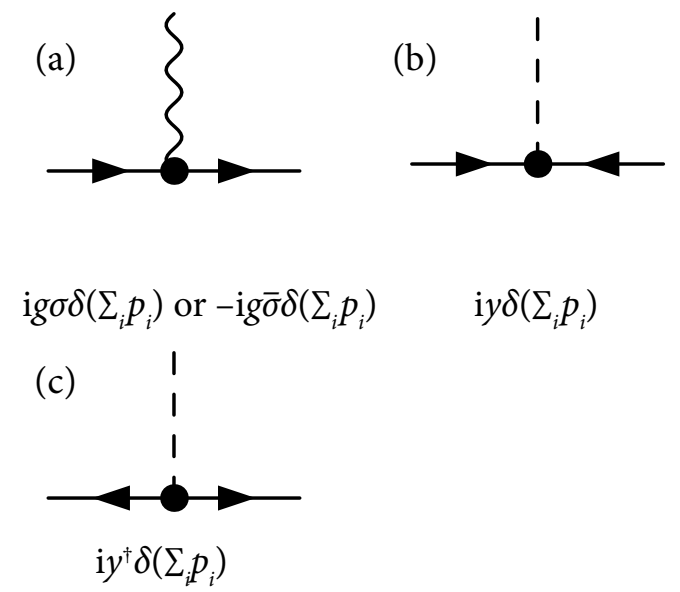

Fig. 4. Feynman diagrams and rules for vertices. The corresponding Feynman rule is written under each diagram. There are two possible rules for a vertex with a vector boson, as shown in Fig. 4(a). All momenta are defined to flow into the vertex, so that $\delta\left(\Sigma_{i} p_{i}\right)$ gives momentum conservation. The rules for vertices with scalars are shown in Fig. 4(b, c). 
The spinor-vector interaction term, shown in Eq. (67), preserves chirality. Hence arrows on the propagator lines must show in the same direction for this coupling. There is also a freedom in choosing the connection: either $\sigma$ or $\bar{\sigma}$, as seen in Eq. (67). These two rules are related by a relative minus sign. The rules, shown in Fig. 4(a), are consistent with this sign convention:

$$
\mathcal{L}_{\text {int }}=+g \xi L \cdot V \xi^{L \dagger}=-g \xi^{L \dagger} \bar{\sigma} \cdot V \xi^{L} .
$$

Note that we also have a freedom in writing the expression for the propagator as shown in Fig. $4(a, b)$. But $\sigma$ can only be connected with $\bar{\sigma}$ and vice versa as discussed in Subsection 2.2. Once we choose a rule for the vertex, we cannot choose the form of the propagator freely anymore. That means, if we choose a vertex as $\sim \bar{\sigma}$, both propagators must be $\sim \sigma$ to form a product $\sim \sigma \bar{\sigma} \sigma$. We illustrate this by an example in the next subsection.

\subsection{Using Feynman rules: loop correction}

To check the consistency of the rules and to present an example of using them, we derive Eq. (84) directly from the diagram shown in Fig. 5(b). This example helps to understand the property in Feynman rules for Weyl spinors that is not apparent in the usual Dirac spinor notation: the one to two correspondence between the diagram and the rules appearing in Fig. 4(a), Fig. 2(a) and Fig. 2(b). We use the same abbreviations for the free field propagators as in Eqs. (74) and (75). Taking the momentum flow from left to right, the rules presented in Fig. 2(b) tell us that we can choose either $\bar{P}(p)$ or $P(-p)$ for each fermion line. The rules for the vertex, shown in Fig. 4 (a), give us the freedom to choose between $\mathrm{i} \sigma g \delta\left(\sum_{i} p_{i}\right)$ and $-\mathrm{i} \bar{\sigma} g \delta\left(\sum_{i} p_{i}\right)$. As noted at the end of the previous subsection, we can connect only barred to unbarred sigmas. We integrate over internal momenta of propagators which use up the delta functions that enforce momentum conservation at each vertex. So we are led to two possible ways to write this correction:

$$
\left.\bar{\Pi}(p)=\bar{P}(p)\left[\int_{k}(\mathrm{ig} \sigma) \bar{P}(p+k) D(k) \text { (ig }\right)\right] \bar{P}(p)
$$

or

$$
\Pi(-p)=P(-p)\left[\int_{k}(-\mathrm{i} g \bar{\sigma}) P(-p-k) D(k)(-\mathrm{i} g \bar{\sigma})\right] P(-p) .
$$

Because $P$ and $\bar{P}$ are odd functions of the momentum, $\Pi$ and $\bar{\Pi}$ are also odd functions. If we recover contracted indices, one can see that the functions $\Pi$ and $\bar{\Pi}$ differ only by the index structure and this in- dex structure is the same as for $P$ and $\bar{P}$, respectively. The diagrams for these functions are presented in Fig. 5. The propagator shown in Fig. 2(b) together with its correction in Fig. 5(b) can be written as

$$
\bar{P}(p)+\bar{\Pi}(p) \text { or }-(P(p)+\Pi(p)),
$$

whereas the diagram in Fig. 5(a) leads to a correction for a propagator shown in Fig. 2(a):

$$
P(p)+\Pi(p) \text { or }-(\bar{P}(p)+\bar{\Pi}(p)) .
$$

The corrections do not spoil the index structure and the properties under $p \rightarrow-p$ for correlation functions, which just means that we managed to consistently define Feynman rules. The freedom of choosing one of two rules for a vertex shown in Fig. 4(a) and for propagators shown in Fig. $2(a, b)$ at one loop order is reflected by the two functions for the same diagram as shown in Fig. 5. This justifies the freedom of choosing one of the two rules for the same propagator shown in Fig. 2(a, b) and for the vertex shown in Fig. $4(a)$ at one loop order. (a)

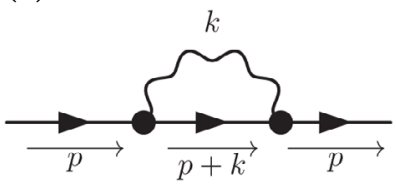

$\Pi(p)$ or $\bar{\Pi}(-p)$ (b)

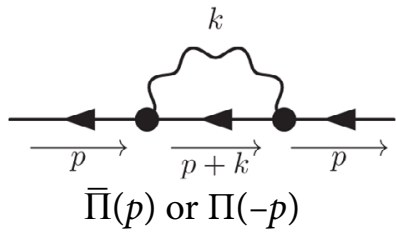

Fig. 5. Gauge loop corrections for the propagators shown in Fig. 2(a, b), respectively.

As discussed in Subsection 3.4.2, the vacuum bubbles do not contribute to the corrections. Also, the tadpole with a gauge boson connected to the propagator gives a vanishing result. The scalar tadpoles shown in Fig. 6 do not vanish. Usually one requires as a renormalization condition that these tadpoles cancel together with the tadpoles and counterterms arriving from corrections to the vacuum expectation value of the scalar field. However, it is important to note that other possibilities in defining renormalization conditions exist and, in principle, tadpoles can also be taken into the definition of a propagator. (a)

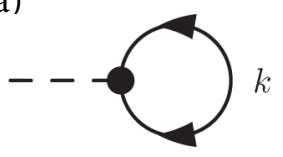

(b)

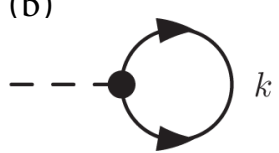

Fig. 6. Tadpole diagrams that give non-vanishing results.

The corrections for a single propagator shown in Fig. 2(a) has four possible forms. This is because there 
are four forms of propagators in the Weyl spinor notation, hence we are led to four possible combinations of external legs shown in Fig. 자.
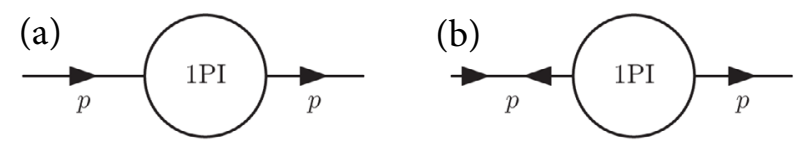

(c)

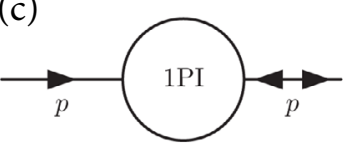

(d)

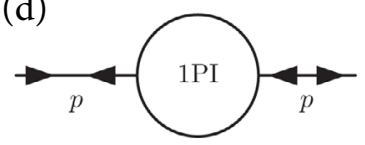

Fig. 7. All possible diagrams for correcting the propagator shown in Fig. 2(a). 1PI stands for the sum of all one particle irreducible diagrams.

\subsection{Using the Feynman rules: example of the Seesaw}

The seesaw mechanism [14-17] is an illustrative example for the usage of the sets of rules for Weyl spinors shown in Figs. 2, 3. Instead of looking at the seesaw extension of the SM, we consider a simplified toy model. We take two Weyl spinors $\xi^{L}$ and $\xi^{R}$ coupled with a Dirac mass term and we give a large Majorana mass to $\xi^{R}$, taking $M \gg m_{\mathrm{D}}$ :

$$
\mathcal{L}_{m}=-\left(m_{\mathrm{D}} \xi^{L} \xi^{R^{\dagger}}+\text { H.c. }\right)-\frac{1}{2} M\left(\xi^{R \dagger} \xi^{R \dagger}+\text { H.c. }\right) \text {. }
$$

We treat the Majorana mass term as a first approximation for the mass of $\xi^{R}$ and the Dirac mass term as a coupling, shown in Fig. 3, which means that to the first approximation $\xi^{L}$ is massless and does not have propagators like in Fig. 2(c, d).

The mass term $m_{\mathrm{D}}$ mixes the fields $\xi^{L}$ and $\xi^{R}$. To estimate the size of this mixing consider the diagram shown in Fig. 3(a), which represents this mixing term. We can interpret this diagram as the field $\xi^{L}$ transforming into $\xi^{R}$ with the coupling of $(-\mathrm{i} m)=\left(-\mathrm{i} m_{D}\right)$. We take the positive momentum direction and assign propagators to external lines for $\xi^{L}$ as in Fig. 2(a) with $m^{2}=0$ and for $\xi^{R}$ as in Fig. 2(b) with $m^{2}=M^{2}$. This correction reads

$$
\frac{\mathrm{i} p \bar{\sigma}}{p^{2}-M^{2}}\left(-\mathrm{i} m_{\mathrm{D}}\right) \frac{\mathrm{i} p \sigma}{p^{2}}=\frac{\mathrm{i} m_{\mathrm{D}}}{p^{2}-M^{2}}=\frac{m_{\mathrm{D}}}{M} \cdot \frac{\mathrm{i} M}{p^{2}-M^{2}},
$$

where we used the property presented in Eq. (18) to get $(p \cdot \bar{\sigma})(p \cdot \sigma)=p^{2}$. Eq. (93) is an expression for the propagator for $\xi^{R}$, of the form shown in Fig. 2(c) with $m=M$ and an additional factor of $\frac{m_{\mathrm{D}}}{M}$. This means that the propagating field $\xi^{L}$ transforms into $\xi^{R}$ by a fraction $\sim \frac{m_{\mathrm{D}}}{M}$.

We further explore diagrams that give corrections to the $\xi^{L}$ propagator. The correction arising from the Dirac mass term for a propagator of $\xi^{L}$ is shown in Fig. 8(a). The diagram of Fig. 8(b) gives rise to a propagator of a form shown in Fig. 2(d) that is absent in the case when the Dirac mass term is neglected. Considering the case, where $\xi^{L}$ is near its mass shell, we have $p^{2} \ll M^{2}$. The diagram in Fig. 8(b), using the rules from Figs. 2, 3 , gives

$$
\begin{aligned}
& \frac{\mathrm{i} p \bar{\sigma}}{p^{2}} \cdot \mathrm{i} m_{\mathrm{D}} \cdot \frac{\mathrm{i} M}{-M^{2}} \cdot \mathrm{i} m_{\mathrm{D}} \cdot \frac{\mathrm{i} p \sigma}{p^{2}} \\
& =-\mathrm{i} \frac{m_{\mathrm{D}}^{2}}{M} \frac{1}{p^{2}} \equiv-\mathrm{i} m_{\xi} \frac{1}{p^{2}}, m_{\xi}=\frac{m_{\mathrm{D}}^{2}}{M},
\end{aligned}
$$

which is a new propagator for $\xi^{L}$. This expression is the first term in the infinite sum of

$$
-\frac{\mathrm{i} m_{\xi}}{p^{2}-m_{\xi}^{2}}=-\frac{\mathrm{i} m_{\xi}}{p^{2}}-\frac{\mathrm{i} m_{\xi}}{p^{2}}\left(-\mathrm{i} m_{\xi}\right) \frac{\mathrm{i} m_{\xi}}{p^{2}}+\ldots,
$$

which we get when considering infinite copies of this diagram. Note that we have an opposite sign to the normal convention for this propagator.

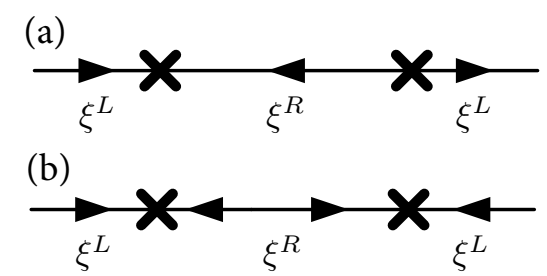

Fig. 8. Mass insertion diagrams for correcting the propagator of $\xi^{L}$.

Taking the diagram shown in Fig. 8(a) and using $p^{2} \ll M^{2}$ we get

$$
\frac{\mathrm{i} p \sigma}{p^{2}} \mathrm{i} m_{\mathrm{D}} \cdot \frac{\mathrm{i} p \bar{\sigma}}{-M^{2}} \mathrm{i} m_{\mathrm{D}} \cdot \frac{\mathrm{i} p \sigma}{p^{2}}=\mathrm{i} \frac{p \sigma}{p^{2}} \frac{\left(\mathrm{i} m_{\mathrm{D}}\right)^{2}}{M^{2}} .
$$

Equivalently, considering the infinite sum of copies of this diagram, one gets

$$
\mathrm{i} \frac{p \sigma}{p^{2}}\left(1+\left(\mathrm{i} \frac{m_{\mathrm{D}}}{M}\right)^{2}+\left(\mathrm{i} \frac{m_{\mathrm{D}}}{M}\right)^{4} \ldots\right)=\mathrm{i} \frac{p \sigma}{p^{2}\left(1+\frac{m_{\mathrm{D}}^{2}}{M^{2}}\right)} .
$$

From this propagator, we see that the field is rescaled by a factor of $\sqrt{1+\frac{m_{\mathrm{D}}^{2}}{M^{2}}}$.

Having the result from Eq. (93), we can define a new field, to include the admixture of $\xi^{R}$ with a fraction of $-\frac{m_{\mathrm{D}}}{M}$ :

$$
\xi_{\text {new }}^{L}=\xi^{L}-\frac{m_{\mathrm{D}}}{M} \xi^{R^{\dagger}} .
$$


Then the transformation for $\xi^{R \dagger}$ reads

$$
\xi_{\text {new }}^{{ }^{\dagger}}=\frac{m_{\mathrm{D}}}{M} \xi^{L}+\xi^{R \dagger} \text {. }
$$

These fields are normalized up to the first order in $\frac{m_{\mathrm{D}}}{M}$. This is evident from Eq. (97), which says that $\xi^{L}$ rescales with a factor of

$$
\sqrt{1+\frac{m_{\mathrm{D}}^{2}}{M^{2}}}=1+O\left(\frac{m_{\mathrm{D}}^{2}}{M^{2}}\right) \text {. }
$$

One can also check that inserting these redefinitions leaves the kinetic term unchanged up to the first order in $\frac{m_{\mathrm{D}}}{M}$. Inserting the inverse transformation

$$
\begin{aligned}
& \xi^{L}=\xi_{\text {new }}^{L}+\frac{m_{\mathrm{D}}}{M} \xi_{\text {new }}^{R \dagger}, \\
& \xi^{R \dagger}=-\frac{m_{\mathrm{D}}}{M} \xi_{\text {new }}^{L}+\xi_{\text {new }}^{R \dagger}
\end{aligned}
$$

into Eq. (92) we get

$$
\begin{aligned}
& \mathcal{L}_{m}=-\frac{1}{2}\left(-\frac{m_{\mathrm{D}}^{2}}{M} \xi_{\text {new }}^{L} \xi_{\text {new }}^{L}+H . c .\right) \\
& -\frac{1}{2} M\left(\xi_{\text {new }}^{R \dagger} \xi_{\text {new }}^{R \dagger}+\text { H.c. }\right)+O\left(\frac{m_{\mathrm{D}}^{2}}{M^{2}}\right) .
\end{aligned}
$$

We see that the phase of $\xi_{\text {new }}^{L}$ should be redefined in order to get the right sign for the mass term. This redefinition of the phase to get a positive mass term in the Lagrangian also cancels the minus sign in Eq. (95), which means that we recover the normal convention for a propagator. The phase of the parameter $\frac{m_{D}^{2}}{M}$ can also be absorbed into the field definition. So the final mass term for the redefined fields can be written as

$$
\begin{aligned}
& \mathcal{L}_{m}=-\frac{1}{2} m_{\xi}\left(\xi_{\text {new }}^{L} \xi_{\text {new }}^{L}+\text { H.c. }\right) \\
& -\frac{1}{2} M\left(\xi_{\text {new }}^{R \dagger} \xi_{\text {new }}^{R \dagger}+\text { H.c. }\right)+O\left(\frac{m_{\mathrm{D}}^{2}}{M^{2}}\right),
\end{aligned}
$$

where $m_{\xi}$ and $M$ are real Majorana masses. By these redefinitions, we get rid of the mixing between the two spinors up to the first order in $\frac{m_{\mathrm{D}}}{M}$. The mass parameter $m_{\xi}$ is the same as in Eq. (94).

\section{Conclusions}

The main confusion in using Feynman rules in the Weyl spinor notation comes from keeping track of definitions. We see that in the Weyl spinor formulation we have an additional freedom of choosing between two equivalent rules for the same diagram.
This one-to-two correspondence between diagrams and rules, as we see in Figs. 2 and 4, makes it even more complicated to follow where minus signs must appear. We try to ease this confusion by presenting explicit derivations of Feynman rules from the path integral and emphasizing on the definitions. We also define propagators in the momentum space rather than in the position space. This leads to the unusual looking propagator definitions presented in Eqs. (51) to (54). Concentrating on the momentum space we explore different choices of momentum dependences of the fields: Majorana spinors do not have the freedom in choosing momentum signs in the Fourier transformation, whereas the Dirac spinors do. In order to have the same definition for both cases, we introduce the convention to fix the momentum dependences of the Dirac spinor.

The examples presented here, loop corrections and the seesaw mechanism, are related to our future work. We plan to explore the nature of Weyl spinors with mixed mass terms in broken gauge field theories. The Standard Model with the seesaw mechanism for one family will be our next step. Later we will include mixings between families and a richer Higgs sector than in the Standard Model. The mixing terms then complicate the analysis and Weyl spinors, as the smallest representation for fermions can show their full advantage over the usual 4-component spinor notation.

The authors thank the Lithuanian Academy of Sciences for the support (Project DaFi2015).

\section{References}

[1] K.M. Heeger, Evidence for neutrino mass: A decade of discovery, in: SEASAW 25: Proceedings of the International Conference on the Seesaw Mechanism, eds. J. Orloff, S. Lavignac, and M. Cribier (World Scientific, Singapore, 2005) pp. 65-80, https://arxiv.org/abs/hep-ex/0412032

[2] SEESAW 25: Proceedings of the International Conference on the Seesaw Mechanism, eds. J. Orloff, S. Lavignac, and M. Cribier (World Scientific, Singapore, 2005).

[3] M.E. Peskin and D.V. Schroeder, An Introduction to Quantum Field Theory (Addison-Wesley, Reading, USA, 1995), http://www.slac.stanford.edu/spires find/books/www? cl=QC174.45\%3AP4

[4] I.J.R. Aitchison and A.J.G. Hey, Gauge Theories in Particle Physics: A Practical Introduction. Volume 1: From Relativistic Quantum Mechanics to QED (IOP, Bristol, UK, 2003), http://www-spires.fnal.gov/ spires/find/books/www?cl=QC793.3.F5A34::2012

[5] H.K. Dreiner, H.E. Haber, and S.P. Martin, Twocomponent spinor techniques and Feynman 
rules for quantum field theory and supersymmetry, Phys. Rept. 494, 1-196 (2010), http://dx.doi. org/10.1016/j.physrep.2010.05.002

[6] A. Bilal, Introduction to Supersymmetry, lecture notes (2001), https://arxiv.org/abs/hep-th/0101055

[7] S. Dittmaier, Weyl-van der Waerden formalism for helicity amplitudes of massive particles, Phys. Rev. D 59, 016007 (1998), http://dx.doi.org/10.1103/ PhysRevD.59.016007

[8] J.B. Albert, D.J. Auty, P.S. Barbeau, D. Beck, V. Belov, M. Breidenbach, T. Brunner, A. Burenkov, G.F. Cao, C. Chambers, et al. (EXO-200 Collaboration), Search for Majorana neutrinos with the first two years of EXO-200 data, Nature 510, 229-234 (2014), http://dx.doi.org/10.1038 nature13432

[9] G. Senjanovic, Neutrino mass: From LHC to grand unification, Riv. Nuovo Cim. 34, 1-68 (2011), http://dx.doi.org/10.1393/ncr/i2011-10061-8

[10] H. Weyl, Electron and gravitation. 1, Z. Phys. 56, 330-352 (1929) [in German], http://dx.doi. org/10.1007/BF01339504
[11]P.B. Pal, Dirac, Majorana and Weyl fermions, Am. J. Phys. 79, 485-498 (2011), http://dx.doi. org/10.1119/1.3549729

[12]T. Ohlsson, Relativistic Quantum Physics: From Advanced Quantum Mechanics to Introductory Quantum Field Theory (Cambridge University Press, Cambridge, UK, 2011).

[13] M. Srednicki, Quantum Field Theory (Cambridge University Press, Cambridge, UK, 2007).

[14] P. Minkowski, $\mu \rightarrow e \gamma$ at a rate of one out of $10^{9}$ muon decays? Phys. Lett. B 67, 421-428 (1977), http://dx.doi.org/10.1016/0370-2693(77)90435-X

[15] R.N. Mohapatra and G. Senjanovic, Neutrino mass and spontaneous parity violation, Phys. Rev. Lett. 44, 912 (1980), http://dx.doi.org/10.1103/ PhysRevLett.44.912

[16] T. Yanagida, Horizontal symmetry and masses of neutrinos, Conf. Proc. C 7902131, 95-99 (1979).

[17]M. Gell-Mann, P. Ramond, and R. Slansky, Complex spinors and unified theories, Conf. Proc. C 790927, 315-321 (1979).

\title{
FEINMANO TAISYKLĖS VEILIO SPINORIAMS SU SUMAIŠYTAIS DIRAKO IR MAJORANOS MASĖS NARIAIS
}

\author{
V. Dūdènas, T. Gajdosik \\ Vilniaus universiteto Teorines fizikos katedra, Vilnius, Lietuva
}

\section{Santrauka}

Pristatome formalizmą, reikalingą norint naudoti Veilio spinorius remiantis Feinmano taisyklemis. Pagrindinis demesys skiriamas Veilio spinoriams, sumaišytiems su Dirako ir Majoranos masès nariais. Tam, kad būtų aiškūs visi naudojami apibrèžimai, mes išvedame Feinmano taisykles iš trajektorijų integralo. Taip pat pristatome du paprastus Veilio spinorių naudojimo pavyzdžius: fermiono propagatoriaus kilpos pataisų integralų sukonstravimą ir žaislinio sūpuoklių modelio pirmojo artinio masès narių išvedimą. 